\title{
Electroweak skyrmions in the HEFT
}

\author{
Juan Carlos Criado, Valentin V. Khoze and Michael Spannowsky \\ Institute for Particle Physics Phenomenology, Department of Physics, Durham University, \\ Durham, DH1 3LE, U.K. \\ E-mail: juan.c.criado@durham.ac.uk, valya.khoze@durham.ac.uk, \\ michael.spannowsky@durham.ac.uk
}

ABSTRACT: We study the existence of skyrmions in the presence of all the electroweak degrees of freedom, including a dynamical Higgs boson, with the electroweak symmetry being non-linearly realized in the scalar sector. For this, we use the formulation of the Higgs Effective Field Theory (HEFT). In contrast with the linear realization, a well-defined winding number exists in HEFT for all scalar field configurations. We classify the effective operators that can potentially stabilize the skyrmions and numerically find the region in parameter spaces that support them. We do so by minimizing the static energy functional using neural networks. This method allows us to obtain the minimal-energy path connecting the vacuum to the skyrmion configuration and calculate its mass and radius. Since skyrmions are not expected to be produced at colliders, we explore the experimental and theoretical bounds on the operators that generate them. Finally, we briefly consider the possibility of skyrmions being dark matter candidates.

Keywords: Solitons Monopoles and Instantons, Effective Field Theories, Beyond Standard Model, Higgs Physics

ArXiv EPrint: 2109.01596 


\section{Contents}

1 Introduction $\quad 1$

2 Theory 2

$\begin{array}{lll}3 & \text { Skyrmion configurations and energy landscape } & 6\end{array}$

3.1 The energy functional in the spherical ansatz 6

$\begin{array}{ll}3.2 & \text { The Skyrme term }\end{array}$

3.3 Skyrmion stabilisation from other operators in HEFT 8

4 Phenomenology 11

$\begin{array}{ll}4.1 \text { Collider signals } & 11\end{array}$

$\begin{array}{lll}4.2 & \text { Positivity bounds } & 13\end{array}$

4.3 Dark matter 14

5 Conclusions $\quad 14$

$\begin{array}{ll}\text { A Numerical method } & 15\end{array}$

\section{Introduction}

Skyrmions are extended field configurations that behave as new particle degrees of freedom. Initially, they were proposed as a description of baryons within an Effective Field Theory (EFT) description of strong interactions containing only the pion fields [1-3]. Since the pions can be viewed as pseudo-Goldstone bosons arising from the breaking of an $\mathrm{SU}(2)$ symmetry, the original setting can be directly applied to the electroweak sector, in the limit in which the Higgs field is infinitely massive, and the gauge bosons are decoupled, so that only the SU(2) would-be Goldstone bosons are present [4]. Electroweak skyrmions have been shown to survive under certain conditions in more realistic settings, in which these limits are partially removed, which can destroy the topological protection they enjoyed in the first place [5].

The purpose of ref. [6] and of this work is to consider skyrmions in the full electroweak theory, including the effects of both the gauge fields and a dynamical Higgs boson. Skyrmions are unstable when only the Standard Model (SM) interactions are present, but they can be stabilized by including higher-order effective operators. Since the discovery of the Higgs [8, 9], two effective descriptions of the electroweak sector have emerged: the Standard Model EFT (SMEFT), in which the scalars furnish a linear representation of the electroweak symmetry group; and the Higgs Effective Field Theory (HEFT), in which the realization of this symmetry is non-linear. The SMEFT version is studied in ref. [6]. In 
this paper, we focus on the HEFT framework, which we find to be better suited for the description of skyrmions because of the non-trivial topology of its scalar sector.

In section 2, we briefly introduce the relevant sector of the HEFT, discuss the differences with the SMEFT and with the approximations that have been previously taken, and introduce the topological numbers that characterize the topology of its field configurations. In section 3, we study the existence of skyrmions numerically in the presence of the different combinations of HEFT operators. In section 4, we consider the phenomenological consequences of skyrmions and the operators that generate them. This allows us to obtain constraints on the parameter space, in which we include positivity bounds. We summarize our conclusions in section 5 .

\section{Theory}

The relevant degrees of freedom for skyrmions in the electroweak sector are the SU(2) gauge bosons $W_{\mu}^{a}$, the would-be Goldstone bosons $G^{a}$, and the Higgs boson $h$. We neglect the effects of the $\mathrm{U}(1)_{Y}$ gauge sector. In the HEFT formulation, the spontaneously broken $\mathrm{SU}(2)$ symmetry is realized non-linearly through the Callan-Coleman-Wess-Zumino construction $[10,11]$, in which the Goldstones are collected into the unitary matrix

$$
U=\exp \frac{i \sigma^{a} G^{a}}{\sqrt{2} v} \in \mathrm{SU}(2) .
$$

This framework was first applied to the electroweak sector without a Higgs boson [12]. The Higgs can then be introduced as an extension of this EFT with a SU(2) singlet field, having no relation to the Goldstones [13-17]. ${ }^{1}$ The effective Lagrangian is written as

$$
\mathcal{L}=\sum_{i} F_{i}(h / v) \mathcal{Q}_{i}, \quad F_{i}(\eta)=\sum_{n=0}^{\infty} c_{i, n} \eta^{n},
$$

where the $\mathcal{Q}_{i}$ are monomials in $W_{\mu \nu}, U, h$ and their covariant derivatives, with $h$ appearing only through its derivatives. That is, schematically

$$
\mathcal{Q}_{i} \sim h^{\mathrm{H}_{i}}\left(W_{\mu \nu}\right)^{\mathrm{W}_{i}} U^{\mathrm{U}_{i}} D^{\mathrm{D}_{i}},
$$

where $\mathrm{H}_{i}, \mathrm{~W}_{i}, \mathrm{D}_{i}$ are, respectively, the number of Higgs fields, field-strength tensors, and covariant derivatives contained in $\mathcal{Q}_{i}$. We use the convention in which the gauge coupling $g$ is absorbed in the gauge fields, with the coefficient of the gauge kinetic term being $-1 /\left(2 g^{2}\right)$. In this setting, the chiral dimension $d_{\chi}$ is defined as [18]

$$
d_{\chi}(h)=d_{\chi}(U)=0, \quad d_{\chi}(W)=2 d_{\chi}(D)=2 .
$$

We adopt a power counting based on the chiral dimension, in which each $c_{i, n}$ coefficient is of order $\Lambda^{2-d_{\chi}\left(\mathcal{Q}_{i}\right)}$, where $\Lambda$ is the HEFT cut-off scale, multiplied by the necessary power of

\footnotetext{
${ }^{1}$ This is to be contrasted with the more restrictive linear realization where $h(x)$ and $U(x)$ are assembled into the Higgs doublet $\phi=\frac{1}{\sqrt{2}}(v+h) U\left(\begin{array}{l}0 \\ 1\end{array}\right)$.
} 


\begin{tabular}{|ccc|}
\hline Name & Operator & Radial energy density $\rho_{i}$ in spherical ansatz \\
\hline $\mathcal{Q}_{1}$ & $\lambda$ & $-\frac{r^{2}}{e^{2} v^{4}}$ \\
$\mathcal{Q}_{h}$ & $\partial_{\mu} h \partial^{\mu} h$ & $\frac{r^{2}}{2}\left(\eta^{\prime}\right)^{2}$ \\
$\mathcal{Q}_{U}$ & $\left\langle D_{\mu} U^{\dagger} D^{\mu} U\right\rangle$ & $\frac{2}{v^{2}}\left(f_{1}^{2}+f_{2}^{2}+\frac{r^{2}}{2} b^{2}\right)$ \\
$\mathcal{Q}_{X h 2}$ & $\frac{1}{g^{2}}\left\langle W_{\mu \nu} W^{\mu \nu}\right\rangle$ & $-8 e^{2}\left[\left(f_{1}^{\prime}-2 b f_{2}\right)^{2}+\left(f_{2}^{\prime}+\left(2 f_{1}-1\right) b\right)^{2}+\frac{2}{r^{2}}\left(f_{1}^{2}+f_{2}^{2}-f_{1}\right)^{2}\right]$ \\
$\mathcal{Q}_{X h 5}$ & $\epsilon^{\mu \nu \rho \sigma}\left\langle W_{\mu \nu} W_{\rho \sigma}\right\rangle$ & 0 \\
$\mathcal{Q}_{X U 8}$ & $i\left\langle W_{\mu \nu}\left[L^{\mu}, L^{\nu}\right]\right\rangle$ & $\frac{16 e^{2}}{2 r^{2}}\left[\left(f_{1}^{2}+f_{2}^{2}\right)\left(f_{1}^{2}+f_{2}^{2}-f_{1}+2 r^{2} b^{2}\right)-b r^{2}\left(f_{2} f_{1}^{\prime}-f_{1} f_{2}^{\prime}+b f_{1}\right)\right]$ \\
$\mathcal{Q}_{X U 11}$ & $i \epsilon^{\mu \nu \rho \sigma}\left\langle W_{\mu \nu}\left[L_{\rho}, L_{\sigma}\right]\right\rangle$ & 0 \\
$\mathcal{Q}_{D 1}$ & $\left\langle L_{\mu} L^{\mu}\right\rangle^{2}$ & $-\frac{4 e^{2}}{r^{2}}\left[2\left(f_{1}^{2}+f_{2}^{2}\right)+r^{2} b^{2}\right]^{2}$ \\
$\mathcal{Q}_{D 2}$ & $\left\langle L_{\mu} L_{\nu}\right\rangle\left\langle L^{\mu} L^{\nu}\right\rangle$ & $-\frac{4 e^{2}}{r^{2}}\left[2\left(f_{1}^{2}+f_{2}^{2}\right)^{2}+r^{4} b^{4}\right]$ \\
$\mathcal{Q}_{D 7}$ & $\left\langle L_{\mu} L^{\mu}\right\rangle \partial_{\nu} h \partial^{\nu} h$ & $-e^{2} v^{2}\left(\eta^{\prime}\right)^{2}\left[2\left(f_{1}^{2}+f_{2}^{2}\right)+r^{2} b^{2}\right]$ \\
$\mathcal{Q}_{D 8}$ & $\left\langle L_{\mu} L_{\nu}\right\rangle \partial^{\mu} h \partial^{\nu} h$ & $-e^{2} v^{2}\left(\eta^{\prime}\right)^{2} r^{2} b^{2}$ \\
$\mathcal{Q}_{D 11}$ & $\left(\partial_{\mu} h \partial^{\mu} h\right)^{2}$ & $-\frac{e^{2} v^{4}}{4}\left(\eta^{\prime}\right)^{4} r^{2}$ \\
\hline
\end{tabular}

Table 1. Custodial-invariant operators $\mathcal{Q}_{i}$ containing the Higgs only through derivatives, of order up to $\Lambda^{0}$, together with their contribution to the radial energy density $\rho_{i}$ in the spherical ansatz, defined in eq. (3.2).

$v$ for the coefficient to have the correct energy dimensions. Thus, terms with higher chiral dimensions are suppressed by higher powers of $v / \Lambda$. An upper bound of $\Lambda \lesssim 4 \pi v$ can be obtained by requiring that the coefficients are not finely tuned [19]. In addition, $\Lambda$ must be large enough for pertubation theory in the effective parameter $v / \Lambda$ to work. A rough estimate of the corresponding lower bound on $\Lambda$ is $v$. Thus, $\Lambda$ must lie in the range:

$$
v<\Lambda \lesssim 4 \pi v
$$

In order for the power counting to hold for the SM terms, the EW coupling constant $g$ and Higgs quartic coupling $\lambda$ are absorbed into the corresponding $\mathcal{Q}_{i}$ operators, and assigned a chiral dimension of

$$
2 d_{\chi}(g)=d_{\chi}(\lambda)=2 .
$$

We keep terms with chiral dimension up to 4 , and impose custodial symmetry, which is needed for configurations in the spherical ansatz to give spherically symmetric contributions to the energy, as described in section 3.1. A list of all relevant operators $\mathcal{Q}_{i}$ is given in table 1 , partially following the notation of ref. [17], where angle brackets $\langle\cdot\rangle$ denote a trace and $L_{\mu}=i U D_{\mu} U^{\dagger}$.

The relevant sector of the SM Lagrangian is given by the $d_{\chi}=2$ operators, with

$$
\begin{aligned}
& F_{1}(h / v)=\frac{1}{\lambda} V(h)=v^{4}\left((h / v)^{2}+(h / v)^{3}+\frac{1}{4}(h / v)^{4}\right)=v^{2} h^{2}+v h^{3}+\frac{h^{4}}{4}, \\
& F_{h}(h / v)=\frac{1}{2}, \quad F_{X h 2}(h / v)=-\frac{1}{2}, \quad F_{U}(h / v)=\frac{v^{2}}{4}\left(1+\frac{h}{v}\right)^{2},
\end{aligned}
$$


Deviations from the SM are encoded in modifications of any of the $F_{i}$. We look for an embedding of the original Skyrme model [1] in the HEFT. This is given by the Lagrangian $\mathcal{L}_{\mathrm{SM}}+\mathcal{L}_{\mathrm{Sk}}$, where $\mathcal{L}_{S M}$ is the SM Lagrangian and

$$
\mathcal{L}_{\mathrm{Sk}}=-\frac{1}{16 e^{2}}\left(\mathcal{Q}_{D 1}-\mathcal{Q}_{D 2}\right),
$$

that is, setting $F_{D 1}(h / v)=-F_{D 2}(h / v)=-1 /\left(16 e^{2}\right)$. In the chiral dimension power counting, the size of the coefficient is given by $e \sim \Lambda /(4 v) .{ }^{2}$ Examples of UV completions of the HEFT that generate the $\mathcal{Q}_{D 1}$ and $\mathcal{Q}_{D 2}$ operators can be found in ref. [20]. For example, integrating out a heavy SU(2)-singlet antisymmetric tensor field $R_{\mu \nu}$ that describes a spin-1 particle with mass $M$ and has the following interaction Lagrangian:

$$
\mathcal{L}_{R, \text { int }}=i G\left\langle R_{\mu \nu} L^{\mu} L^{\nu}\right\rangle
$$

generates eq. (2.9) with $e=M /(4 \sqrt{2} G)$.

The theory $\mathcal{L}_{\mathrm{SM}}+\mathcal{L}_{\mathrm{Sk}}$ is a candidate for the stabilization of skyrmions. Two limits of it have been previously studied in the literature:

A. Frozen Higgs. This corresponds to $m_{h} \rightarrow \infty$, which implies that the Higgs is set to its vev $h=0$ everywhere.

B. No gauge fields. This is obtained when the $\mathrm{SU}(2)$ coupling vanishes, $g \rightarrow 0$. In this limit, the coefficient of the $\left\langle W_{\mu \nu} W^{\mu \nu}\right\rangle$ term becomes large, and the gauge fields are forced to approach a pure gauge configuration in order to minimize the energy. One can gauge them away. The only degrees of freedom left are the Goldstone bosons and the Higgs.

Taking both limits leads to a theory with only the Goldstone bosons as dynamical degrees of freedom, which has been studied in, e.g. ref. [3]. Limit $\mathbf{A}$ has been considered in ref. [5], while limit $\mathbf{B}$ has been considered in ref. [21]. In any of these limits, and in the full theory, the Skyrme term can be generalized by allowing other linear combinations of the $\mathcal{Q}_{D 1}$ and $\mathcal{Q}_{D 2}$ operators. This has been done in the case where both limits are taken, in ref. [4], and in limit $\mathbf{B}$, in ref. [22].

In ref. [6] skyrmions were studied in the full theory without assuming any of the two limits above. This was done within the SMEFT framework, in which the electroweak symmetry is realized linearly. The purpose of the present paper is to continue this program in the non-linear realization. Ultimately, the existence of skyrmions turns out to be much harder to prove in the SMEFT than in the HEFT, as discussed below. Ref. [23], which appeared during the preparation of this work, has a similar scope.

In limit $\mathbf{B}$, the theory contains stable field configurations separated from the vacuum by an infinite energy barrier. This fact can be understood from a topological point of view. To have finite energy, the fields must approach a pure gauge configuration at spatial

\footnotetext{
${ }^{2}$ Notice that, following eq. (2.5), the allowed range of values for $e$ is $1 / 4<e \lesssim \pi$.
} 
infinity. This means that one can always choose a gauge in which the following boundary conditions are satisfied:

$$
\lim _{|\mathbf{x}| \rightarrow \infty} W_{i}(x)=0, \quad \lim _{|\mathbf{x}| \rightarrow \infty} h(x)=0, \quad \lim _{|\mathbf{x}| \rightarrow \infty} U(x)=1_{2 \times 2},
$$

which means that all directions towards infinity can be identified with a single point, effectively compactifying space into $S^{3}$. Thus, the scalar fields can be viewed as a $S^{3} \rightarrow$ $\mathbb{R} \times S^{3}$ mapping. We can then define a topological charge, the winding number for the $U: S^{3} \rightarrow S^{3}$ part of the mapping:

$$
n_{U}=\frac{1}{24 \pi^{2}} \epsilon_{i j k} \int d^{3} x\left\langle L_{i} L_{j} L_{k}\right\rangle .
$$

This is a homotopy invariant of $U$, and therefore it can never change with smooth time evolution. However, this number is only well defined when the target space of the scalar sector has the topology $\mathbb{R} \times S^{3}$. This is true generically both in the full HEFT and in limit $\mathbf{B}$, but it ceases to be in the particular case of the SMEFT, in which the Lagrangian becomes independent of $U(x)$ when $h(x)=-v$ (see footnote 1 above). One can then identify all points with this value of $h$, turning the scalar manifold into $\mathbb{R}^{4} \cong \mathbb{C}^{2}$. The scalar degrees of freedom are thus collected into a SU(2) doublet $\phi$. In general, the topology of the static configurations of $\phi$ cannot be characterized in terms of the number $n_{U}$ since $U$ is only defined through $\phi=\frac{1}{\sqrt{2}}(v+h) U \cdot(01)^{T}$ when $\phi \neq 0$ everywhere. ${ }^{3}$ Therefore, in order for skyrmions to be stable in the SMEFT, it is necessary that a local minimum of the energy with well-defined $n_{\mathrm{Sk}} \simeq 1$, and thus $\phi \neq 0$, exists. In particular, this field configuration must be separated by a barrier from any other configuration with $\phi(x)=0$ at some point $x$. This property has not been proven for skyrmions in the SMEFT.

One can recover a well-defined $n_{U}$ in the SMEFT by taking the frozen Higgs limit $\mathbf{A}$, which disallows $h(x)=-v$ and forces the scalars to be in the submanifold $S^{3}$. As already noted earlier, we will not follow this route in this paper and will instead use the HEFT formulation of the theory, where $h$ and $U$ are independent, and without imposing limits $\mathbf{A}$ and $\mathbf{B}$.

The inclusion of gauge fields destroys the topological protection of $n_{U} \neq 0$ configurations from decaying into the vacuum. However, the $\left\langle W_{i j} W^{i j}\right\rangle$ term in the energy induces a finite-energy barrier between configurations in which $W_{\mu}$ is a pure gauge, $W_{i}=\mathcal{U} \partial_{i} \mathcal{U}^{\dagger}$, $W_{0}=0$, possibly making them metastable. In order to describe this, we use the ChernSimons number

$$
n_{\mathrm{CS}}=\frac{1}{16 \pi^{2}} \epsilon_{i j k} \int d^{3} x\left\langle W_{i} W_{j k}+\frac{2 i}{3} W_{i} W_{j} W_{k}\right\rangle .
$$

For a pure-gauge $W_{i}=\mathcal{U} \partial_{i} \mathcal{U}^{\dagger}, n_{\mathrm{CS}}$ is the integer winding number of the gauge transformation $\mathcal{U}(\mathbf{x}): S^{3} \rightarrow S^{3}$.

A skyrmion is a field configuration for which $n_{U}$ and $n_{\mathrm{CS}}$ differ by (approximately ${ }^{4}$ ) one unit. We thus define the skyrmion number as

$$
n_{\mathrm{Sk}}=n_{U}-n_{\mathrm{CS}} .
$$

\footnotetext{
${ }^{3}$ Even if $\phi=0$ only at an isolated point $p, U$ becomes a mapping $S^{3}-\{p\} \cong \mathbb{R}^{3} \rightarrow S^{3}$, and all such mappings are homotopically equivalent.

${ }^{4}$ Due to metastability.
} 
While $n_{U}$ and $n_{\mathrm{CS}}$ are not gauge invariant, $n_{\mathrm{Sk}}$ is, because $n_{U}$ and $n_{\mathrm{CS}}$ change by the same integer under a large gauge transformation. An anti-skyrmion is similarly a configuration where $n_{\mathrm{Sk}} \simeq-1$, and multi-skyrmions have $\left|n_{\mathrm{Sk}}\right|>1$. A CP transformation changes the sign of the skyrmion number.

\section{Skyrmion configurations and energy landscape}

\subsection{The energy functional in the spherical ansatz}

We parametrize the space of static configurations of the fields $W_{\mu}^{a}, U$ and $h$ in the $W_{0}=0$ gauge by means of 4 real functions of one variable: $f_{1}, f_{2}, b$ and $\eta$. We do so by further imposing the unitary gauge $U(\mathbf{x})=1_{2 \times 2}$ and the spherical ansatz:

$$
W_{i}(\mathbf{x})=v e \tau_{a}\left(\epsilon_{i j a} n_{j} \frac{f_{1}(r)}{r}+\left(\delta_{i a}-n_{i} n_{a}\right) \frac{f_{2}(r)}{r}+n_{i} n_{a} b(r)\right), \quad h(\mathbf{x})=\frac{v}{\sqrt{2}} \eta(r),
$$

where $\tau_{a}$ are the Pauli matrices, $n_{i}=x_{i} /|\mathbf{x}|, r=v e|\mathbf{x}|$, and $e$ is a parameter we will adjust as a function of Wilson coefficients. The energy density in this ansatz is spherically symmetric when all interactions are invariant under custodial symmetry. ${ }^{5}$ We can then write the energy as

$$
E=\frac{4 \pi v}{e} \int_{0}^{\infty} d r \sum_{i} F_{i}(\eta) \rho_{i}
$$

where the contributions $\rho_{i}$ to the radial energy density of each $\mathcal{Q}_{i}$ operator are given in table 1. Requiring that the energy is finite and that the fields are regular at the origin gives rise to the following boundary conditions:

$$
\begin{array}{r}
f_{1}(0)=f_{1}^{\prime}(0)=f_{2}(0)=f_{2}^{\prime}(0)-b(0)=\eta^{\prime}(0)=0, \\
f_{1}(\infty)=f_{2}(\infty)=b(\infty)=\eta(\infty)=0 .
\end{array}
$$

Since we have fixed the unitary gauge, the skyrmion number is just $n_{\mathrm{Sk}}=-n_{\mathrm{CS}}$. For convenience, we define

$$
n_{W}=\frac{i}{24 \pi^{2}} \epsilon_{i j k} \int d^{3} x\left\langle W_{i} W_{j} W_{k}\right\rangle=\frac{2}{\pi} \int_{0}^{\infty} d r b\left(f_{1}^{2}+f_{2}^{2}\right)
$$

which agrees with $n_{\mathrm{CS}}$ at integer values. Thus, skyrmion and anti-skyrmions will be found at $n_{W} \simeq-1$ and $n_{W} \simeq 1$, respectively. CP symmetry, which takes one into the other, is given here by $f_{1} \rightarrow f_{1}, f_{2} \rightarrow-f_{2}, b \rightarrow-b, \eta \rightarrow \eta$. All the operators we consider are invariant under this transformation. This is because the two operators that violate CP vanish for static field configurations. Thus, the static-configuration energy functional is invariant under $n_{W} \rightarrow-n_{W}$.

\footnotetext{
${ }^{5}$ Indeed, if one takes any $M \in \mathrm{SU}(2)$ and $R$ its representation as a spatial rotation, one has $M W_{i}(\mathbf{x}) M^{\dagger}=$ $R_{i j} W_{j}\left(R^{-1} \mathbf{x}\right)$, so invariance under spatial rotations and under custodial symmetry become equivalent.
} 

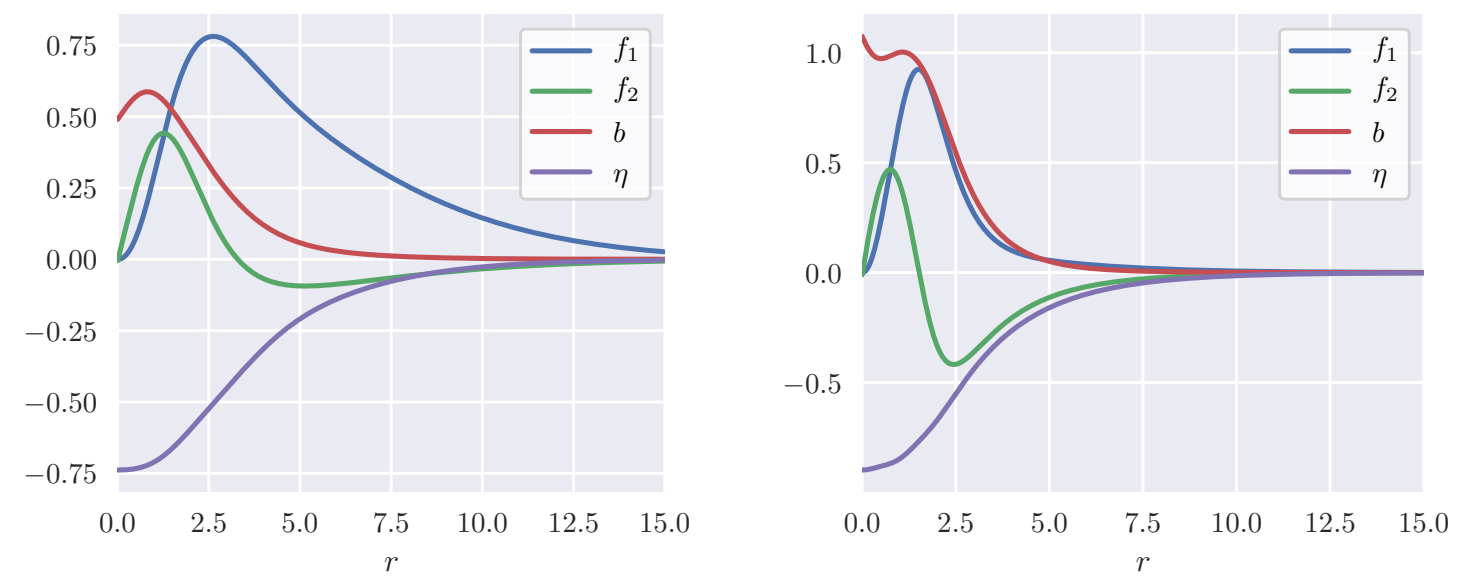

Figure 1. Minimal energy configurations for $e=1.2, c_{D 1,0}=-c_{D 2,0}=1 /\left(16 e^{2}\right)$, and $n_{W}=0.4$ (left) or $n_{W}=0.8$ (right).

\subsection{The Skyrme term}

We focus first on the case in which

$$
-c_{D 1,0}=c_{D 2,0} \equiv \frac{1}{16 e^{2}},
$$

with the rest of non-SM coefficients in the HEFT Lagrangian being set to zero. The last equality is to be understood as fixing the free parameter $e$ of the ansatz. This corresponds to the original Skyrme term, given in eq. (2.9). The total energy functional is given by

$$
E=\frac{4 \pi v}{e} \int_{0}^{\infty} d r\left[\rho_{\mathrm{SM}}+\left(f_{1}^{2}+f_{2}^{2}\right)\left(b^{2}+\frac{f_{1}^{2}+f_{2}^{2}}{2 r^{2}}\right)\right],
$$

where $\rho_{\mathrm{SM}}$ is the contribution from the SM. We shall now describe the field configurations and energy landscape that arise in this setting. We study them using the method described in appendix A. We display two example configurations for $e=1.8$ and different values of $n_{W}$ in figure 1. In figure 2, we show the minimal energy as a function of $n_{W}$, for different values of $e$. We obtain numerically more stable and smoother results than in ref. [6], due the partial topological protection of the HEFT described in section 2. For $e>e_{\text {crit }} \simeq 0.9$, we find a finite-energy barrier separating the skyrmion, with $n_{W} \simeq 1$, and the vacuum at $n_{W}=0$. This barrier disappears below $e_{\text {crit }}$. Thus, the skyrmion solution exists only when $e>e_{\text {crit }}$ and is a metastable configuration.

The energy $E$ of the local minimum is the skyrmion mass. We find that the normalized energy $e M_{\mathrm{Sk}} /(4 \pi v)$ is approximately constant, with a value of 3.3 at $e=e_{\mathrm{crit}}$, and a limiting value of 3 as $e \rightarrow \infty$, so the skyrmion mass is given by

$$
\left.M_{\mathrm{Sk}}\right|_{e \simeq e_{\text {crit }}} \simeq \frac{41 v}{e},\left.\quad M_{\mathrm{Sk}}\right|_{e \rightarrow \infty} \simeq \frac{38 v}{e} .
$$

This is an order of magnitude higher than for the SMEFT solutions found in ref. [6]. The maximum of $M_{\mathrm{Sk}}$ is reached at $e=e_{\mathrm{crit}}$ :

$$
M_{\mathrm{Sk}} \leq\left. M_{\mathrm{Sk}}\right|_{e=e_{\mathrm{crit}}} \simeq 11 \mathrm{TeV} .
$$



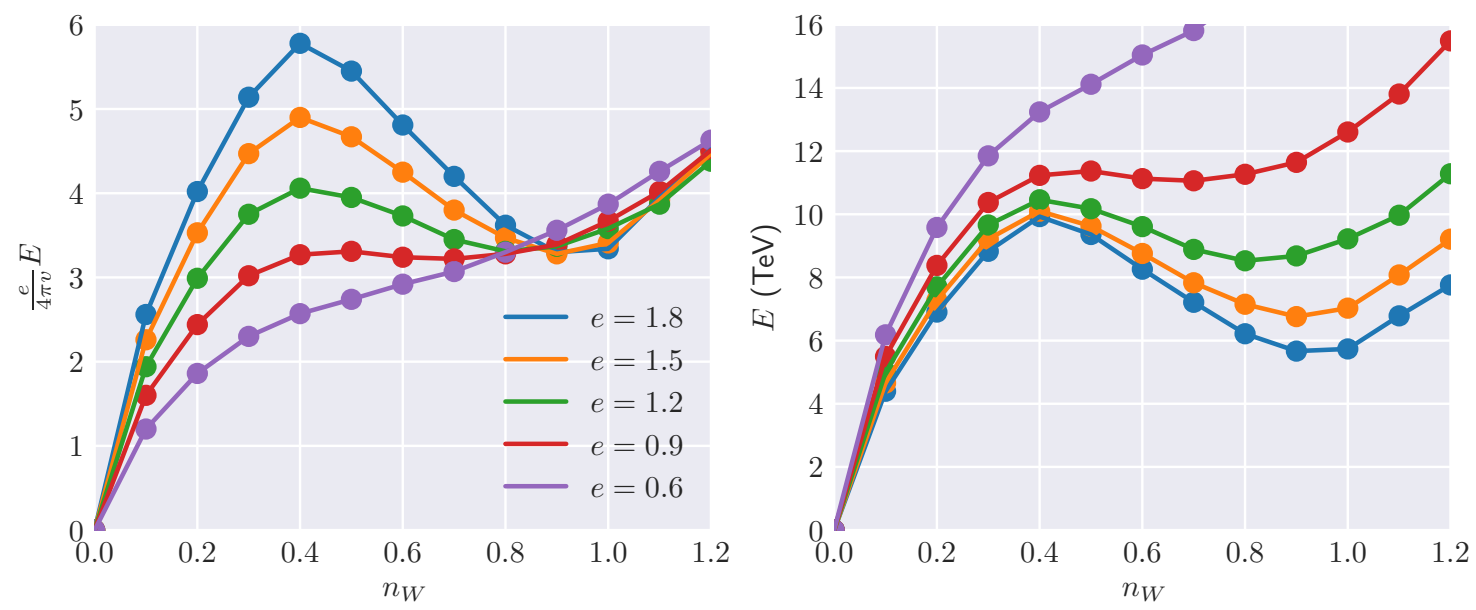

Figure 2. Minimal energy as a function of $n_{W}$, for $c_{D 1,0}=-c_{D 2,0}=1 /\left(16 e^{2}\right)$ and different values of $e$. The barrier disappears around $e=0.9$ and no skyrmion solutions exist at lower values of $e$.

In figure 3, we show this behaviour and compare it to the case in which no gauge fields are present, labelled limit $\mathbf{B}$ in section 2. The curves are similar for large $e$. This is to be expected since a large value of $e$ makes the $\left\langle W_{\mu \nu} W^{\mu \nu}\right\rangle$ term dominant, with similar effects as taking $g \rightarrow 0$, which is limit $\mathbf{B}$. However, some differences arise at small $e$. Just above $e_{\text {crit }}$, the mass of the skyrmion in the full theory is slightly lower than in limit $\mathbf{B}$. This is because the $n_{W}=1$ is no longer topologically fixed in the full theory, and so $n_{W}$ can move to another value with lower energy. For $e<e_{\text {crit }}$, skyrmions become unstable in the full theory, but nothing changes in limit $\mathbf{B}$, as they are still topologically protected.

For the height of the barrier, the energy of the local maximum near $n_{W}=1 / 2$, we find that

$$
\left.E_{\text {barrier }}\right|_{e=e_{\text {crit }}} \simeq 11 \mathrm{TeV}, \quad \text { and }\left.E_{\text {barrier }}\right|_{e \rightarrow \infty} \simeq 10 \mathrm{TeV} .
$$

We also define the radius of the skyrmion $R_{\mathrm{Sk}}$ by averaging over the $n_{W}$ density as

$$
R_{\mathrm{Sk}}^{2}=\frac{i}{24 \pi^{2}} \epsilon_{i j k} \int d^{3} x|\mathbf{x}|^{2}\left\langle W_{i} W_{j} W_{k}\right\rangle=\frac{2}{\pi(v e)^{2}} \int d r r^{2} b\left(f_{1}^{2}+f_{2}^{2}\right) .
$$

We find that

$$
\left.R_{\mathrm{Sk}}\right|_{e=e_{\text {crit }}} \simeq \frac{1.4}{v e},\left.\quad R_{\mathrm{Sk}}\right|_{e \rightarrow \infty} \simeq \frac{1.9}{v e},
$$

\subsection{Skyrmion stabilisation from other operators in HEFT}

We consider here the possibility that skyrmions are stabilized by some operator from table 1 other than $\mathcal{Q}_{D 1}-\mathcal{Q}_{D 2}$. Some of these operators can be discarded for this purpose from general considerations. Derrick's theorem [7] forbids the existence of any solitons when only $\mathcal{Q}_{1}, \mathcal{Q}_{h}$ and $\mathcal{Q}_{U}$ are present. Since $n_{\mathrm{CS}}$ is close to an integer for skyrmions, the gauge fields are close to a pure gauge configuration, and all operators containing a field-strength tensor can also be neglected. There are five remaining operators that can contribute: the $\mathcal{Q}_{D i}$ in table 1. 


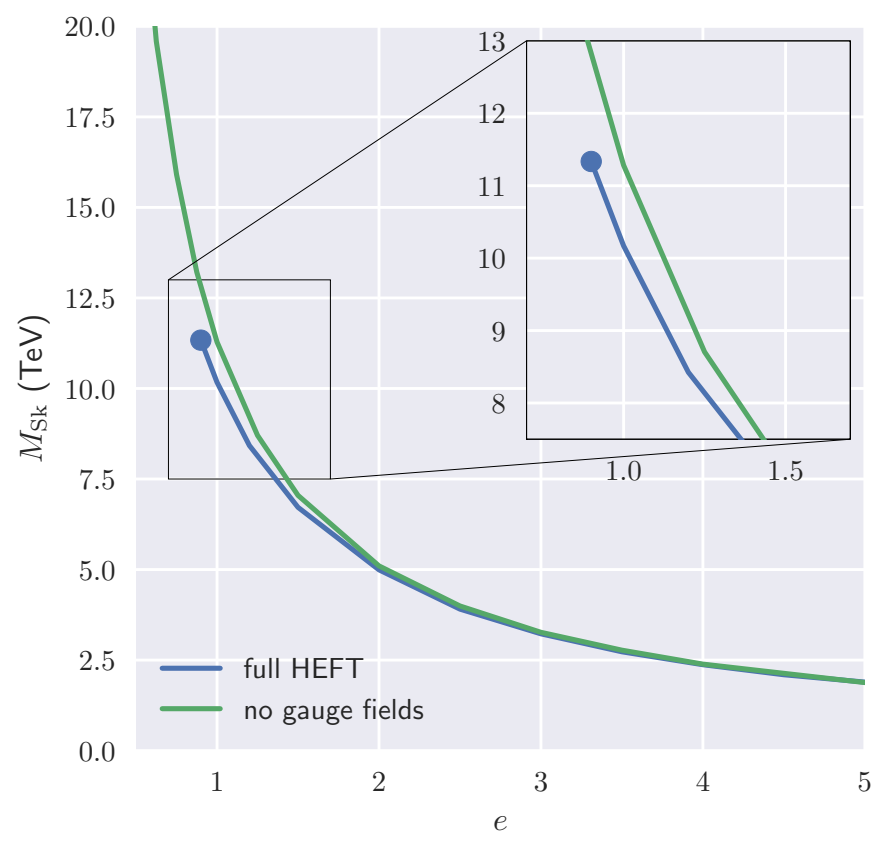

Figure 3. Skyrmion mass $M_{\mathrm{Sk}}$ as a function of $e$ for the full theory and for limit $\mathbf{B}$.

We consider now turning on one $c_{D i, n}$ coefficient at a time while fixing the others to zero. We find that none of them are capable of stabilizing skyrmions except for $c_{D 1,0}$ and $c_{D 2,0}$. Indeed, for all the others, their radial energy density $\rho_{i}$ is multiplied by some monomial in $\eta$ or $\eta^{\prime}$. One can then take $\eta=0$ everywhere, which implies $F_{i}(\eta) \rho_{i}=0$. We have checked this numerically in several examples.

It remains to study the skyrmions generated by $c_{D 1,0}$ and $c_{D 2,0}$. It turns out that both individually, as well as some of their linear combinations, generate meta-stable skyrmions. We parametrize the space of linear combinations with two parameters $e$ and $\theta$, with the former to be used as the corresponding parameter in the spherical ansatz:

$$
c_{D 1,0}=\frac{\sqrt{2}}{16 e^{2}} \cos \theta, \quad c_{D 2,0}=\frac{\sqrt{2}}{16 e^{2}} \sin \theta .
$$

The Skyrme term is recovered for $\theta=3 \pi / 4$. In terms of these parameters, the non-SM contribution to the radial energy reads

$$
c_{D 1,0} \rho_{D 1}+c_{D 2,0} \rho_{D 2}=-\frac{\cos \theta}{4 r^{2}}\left[2\left(f_{1}^{2}+f_{2}^{2}\right) r^{2} b^{2}+2(2+\tan \theta)\left(f_{1}^{2}+f_{2}^{2}\right)^{2}+(1+\tan \theta) r^{4} b^{4}\right]
$$

This is positive everywhere if and only if $\cos \theta \leq 0$ and $\tan \theta \geq-1$, or, equivalently $3 \pi / 4 \leq \theta \leq 3 \pi / 2$. Numerically, we find that skyrmions are stabilized in a slightly wider range: ${ }^{6}$

$$
0.71 \pi \simeq \theta_{\min } \leq \theta \leq \theta_{\max } \simeq 1.6 \pi,
$$

\footnotetext{
${ }^{6}$ The region determined by these values agrees with the one obtained in ref. [4] for the case in which both limit $\mathbf{A}$ and $\mathbf{B}$ are taken.
} 

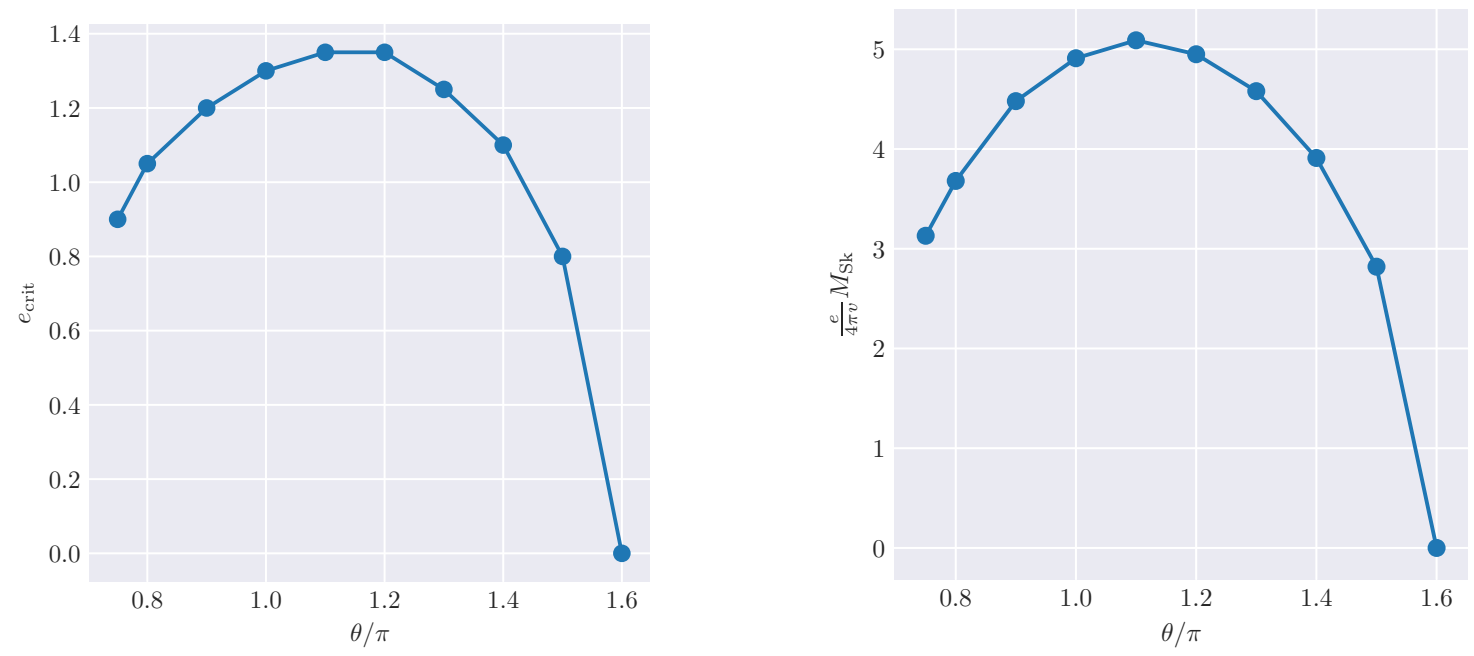

Figure 4. Left: $e_{\text {crit }}$ as a function of $\theta$. Right: skyrmion mass $M_{\mathrm{Sk}}$ as a function of $\theta$, for $e=3$.

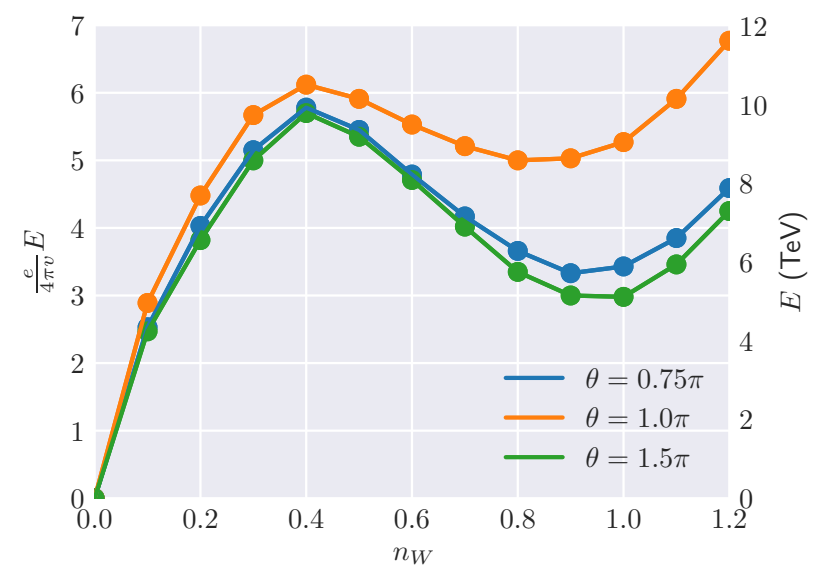

Figure 5. Minimal energy as a function of $n_{W}$, for $e=1.8$ and $\theta=3 \pi / 4, \pi, 3 \pi / 2$.

for $e>e_{\text {crit }}(\theta)$, where $e_{\text {crit }}(\theta)$ is a $\theta$-dependent critical value of $e$, that we show on the left panel in figure 4 . The skyrmion mass also depends on $\theta$ for constant $e$, with $M_{\mathrm{Sk}}=0$ at $\theta=\theta_{\max }$. We show this on the right panel of figure 4 . The normalized mass $e M_{\mathrm{Sk}} /(4 \pi v)$ has little variation with $e$, as it happened for $\theta=3 \pi / 4$. In figure 5 , we display the energy profile for $e=1.8>\max _{\theta} e_{\text {crit }}(\theta)$, and different values of $\theta$. Finally, in figure 6 we show the region of $\left(c_{D 1,0}, c_{D 2,0}\right)$ space where meta-stable skyrmions exist, and the values the masses of the skyrmions inside it, which are given approximately by

$$
M_{\mathrm{Sk}} \simeq(30 \mathrm{TeV}) \cdot\left[\tan \left(\theta_{\max }\right) c_{D 1,0}-c_{D 2,0}\right]^{1 / 2} .
$$

The condition $e>e_{\text {crit }}(\theta)$ is just a $\theta$-independent upper bound on the skyrmion mass $M_{\mathrm{Sk}}<11 \mathrm{TeV}$. The radius is similarly given by

$$
R_{\mathrm{Sk}} \simeq\left(20 \mathrm{TeV}^{-1}\right) \cdot\left[\tan \left(\theta_{\max }\right) c_{D 1,0}-c_{D 2,0}\right]^{1 / 2} \simeq 2 M_{\mathrm{Sk}} / 3 \cdot \mathrm{TeV}^{-2} .
$$




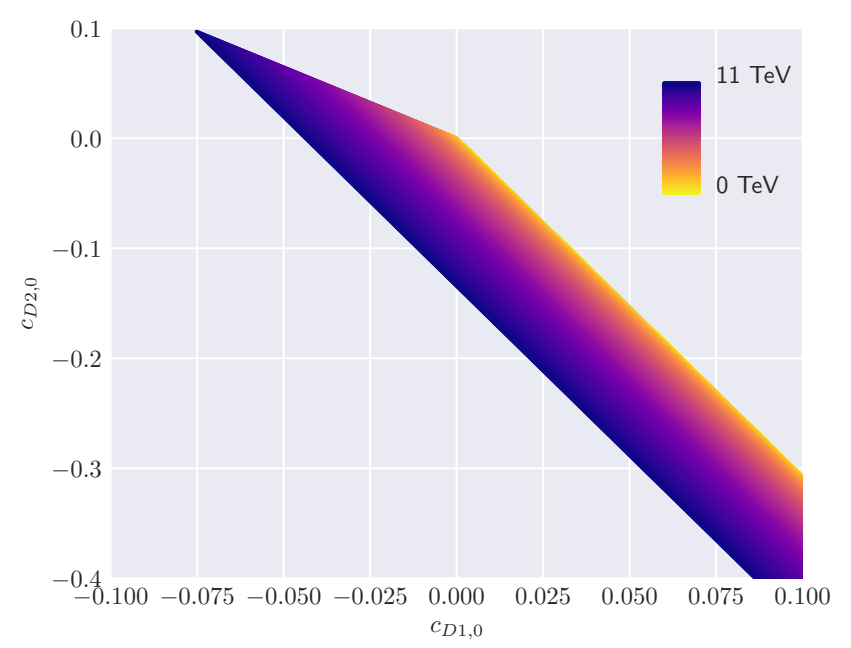

Figure 6. Skyrmion mass $M_{\mathrm{Sk}}$ as a function of $c_{D 1,0}$ and $c_{D 2,0}$.

In order for skyrmions to behave as classical objects, their radius must be larger than their Compton wavelength: $R_{\mathrm{Sk}} \gtrsim 1 / M_{\mathrm{Sk}}$. In combination with the equation above, this implies that $M_{\mathrm{Sk}} \gtrsim 1.2 \mathrm{TeV}$.

The region where skyrmions exist in the $\left(c_{D 1,0}, c_{D 2,0}\right)$ plane is thus determined by

$$
c_{D 2,0}<\tan \left(\theta_{\min }\right) c_{D 1,0}, \quad 1.7 \times 10^{-3} \lesssim \tan \left(\theta_{\max }\right) c_{D 1,0}-c_{D 2,0} \lesssim 0.13
$$

Although the rest of the $c_{i, n}$ coefficients are not enough by themselves to stabilize skyrmions, they may have effects in the configurations generated by $c_{D 1,0}$ and $c_{D 2,0}$. Figure 7 shows the contribution of each $\mathcal{Q}_{i}$ to the energy density in the configuration with $\theta=3 \pi / 4$ and $e=1.8$. The contributions from the operators not included in the generation of the configuration are negligible compared to the energy. This means that whenever the $c_{i, n}$ coefficients are chosen so that their contribution is positive, they will not change the skyrmion configuration in a significant way. However, they might be chosen so that their contribution to the energy is arbitrarily negative, destabilizing the skyrmion. We find numerically that this happens when $c_{D 8,0}=1$, for example.

\section{Phenomenology}

\subsection{Collider signals}

The process of electroweak skyrmion production is similar to the electroweak instanton, as it is a $B+L$ violating transition over a barrier of a few $\mathrm{TeV}$. As such, it is expected to be exponentially suppressed, even at energies above the potential barrier [24, 25]. Thus, it is unlikely that this process will take place at colliders. However, one can indirectly study the existence of skyrmions through other effects of the operators that generate them.

The two skyrmion-stabilizing operators $\mathcal{Q}_{D 1}$ and $\mathcal{Q}_{D 2}$ induce an anomalous quartic gauge coupling (aQGC) while preserving the SM triple gauge coupling. Most LHC searches 

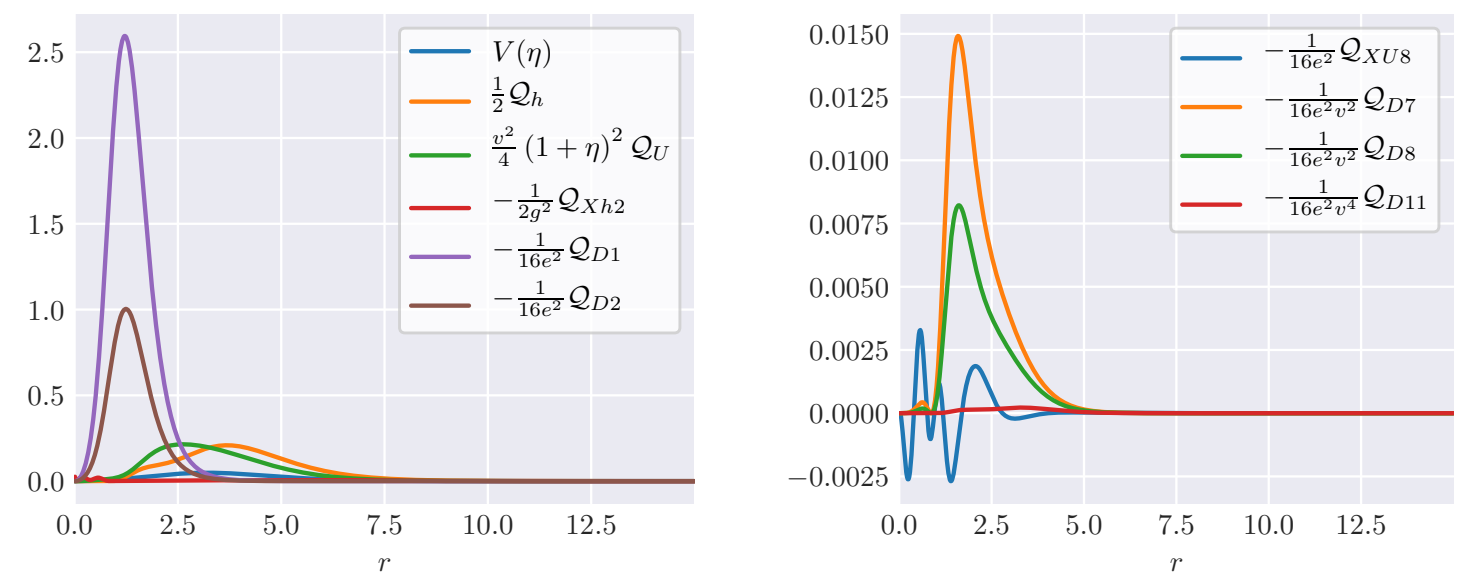

Figure 7. Radial energy densities $\rho_{i}$ in the skyrmion configuration with $\theta=3 \pi / 4$ and $e=1.8$. The operators in the left plot are included in the calculation of the skyrmion configuration. The ones in the right plot are computed once this configuration is obtained and fixed.

for aQGC [26-32] use a parametrization in terms of dimension-8 SMEFT operators which was first proposed in ref. [33]. This set of operators was corrected in ref. [34] by introducing missing operators and removing redundant ones in order for them to form a basis. The space of operators with four covariant derivatives was shown to have dimension 3 . However, the experimental searches with the strongest constraint on this space $[30,31]$ give their results in terms of only two operators, coming from an incomplete set of ref. [33]:

$$
\mathcal{L}_{S}=f_{S 0}\left(D_{\mu} \phi^{\dagger} D_{\nu} \phi\right)\left(D^{\mu} \phi^{\dagger} D^{\nu} \phi\right)+f_{S 1}\left(D_{\mu} \phi^{\dagger} D^{\mu} \phi\right)\left(D_{\nu} \phi^{\dagger} D^{\nu} \phi\right)
$$

Therefore, their results cannot be used in general to constrain the full 3-dimensional space of Wilson coefficients. Only when the measured final state uniquely selects one aQCG vertex ( $W W W W, W W Z Z$ or $Z Z Z Z$ ) can the results in the incomplete set be translated into the complete EFT basis, as shown in ref. [35]. Following this reference, we obtain limits over $c_{D 1,0}$ and $c_{D 2,0}$ (denoted $\alpha_{5}$ and $\alpha_{4}$ there) from the 95\% CL limits over $f_{S 0}$ and $f_{S 1}$ found in ref. [31] individually for $W W$ and $W Z$ production at $\sqrt{s}=13 \mathrm{TeV}$ and $\int L d t=137 \mathrm{fb}^{-1}$. $W W$ production comes from the $W W W W$ vertex, and the limits and conversion are given by

$$
\begin{array}{r}
-2.7 \times 10^{-3} \leq 2 c_{D 1,0}+c_{D 2,0}=\frac{v^{4} f_{S 1}}{8} \leq 2.9 \times 10^{-3}, \\
-8.2 \times 10^{-3} \leq c_{D 2,0}=\frac{v^{4} f_{S 0}}{8} \leq 8.9 \times 10^{-3},
\end{array}
$$

whereas $W Z$ production comes from the $W W Z Z$ vertex, they are

$$
\begin{aligned}
& -1.3 \times 10^{-3} \leq c_{D 1,0}=\frac{v^{4} f_{S 1}}{16} \leq 1.3 \times 10^{-3}, \\
& -1.9 \times 10^{-3} \leq c_{D 2,0}=\frac{v^{4} f_{S 0}}{16} \leq 1.9 \times 10^{-3} .
\end{aligned}
$$




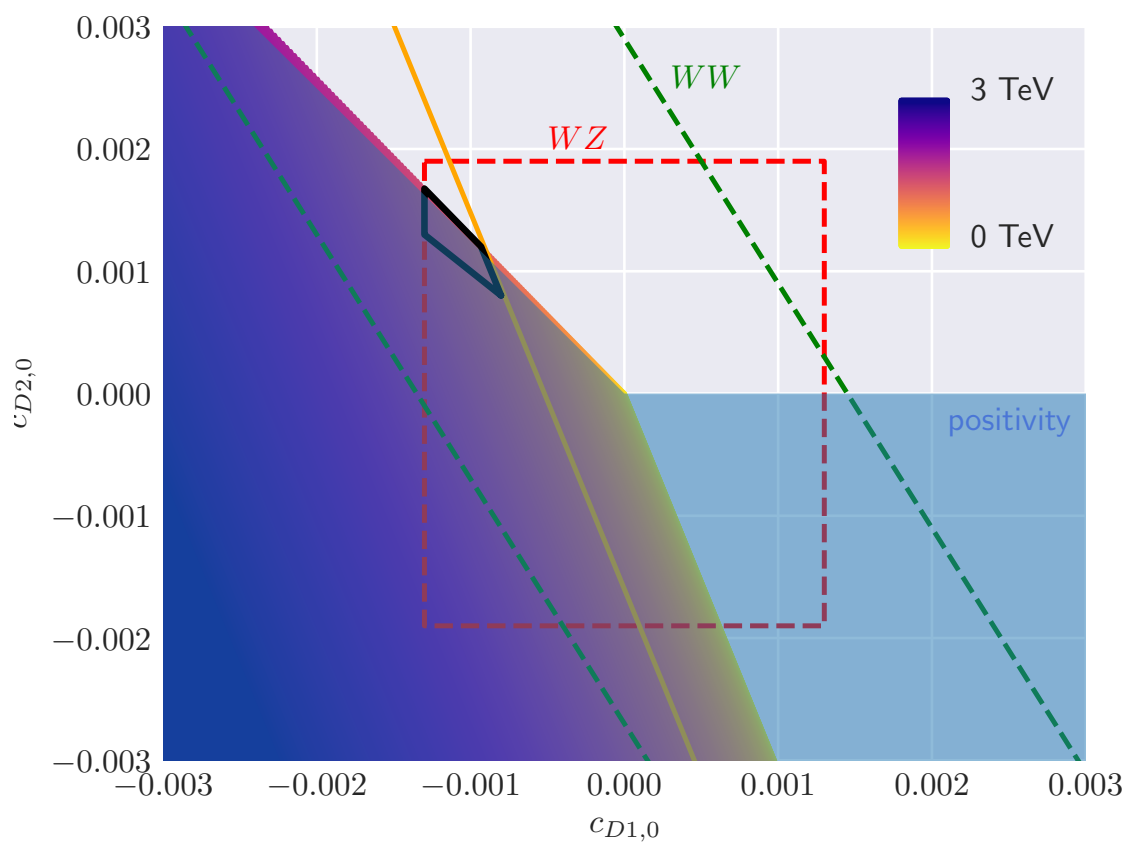

Figure 8. Red and green dashed lines: 95\% CL limits on $c_{D 1,0}$ and $c_{D 2,0}$ at $\sqrt{s}=13 \mathrm{TeV}$, $\int d t L=35.9 \mathrm{fb}^{-1}$ from CMS [30], using data from $W Z$ production (red) and $W W$ production (green). Solid yellow line: lower limit on the skyrmion mass from requiring a classical behavior. Transparent shaded blue region: excluded by positivity bounds. Color-gradient region: allowed values of the coefficients for the existence of skyrmions, from the numerical calculations in this work. The coloring represents the skyrmion mass. The black-line perimeter encloses the region of allowed values of the coefficients that support a skyrmion.

We show these limits in figure 8. We point out that the experimental bounds in ref. [36] are presented in terms of a basis for 2-dimensional custodial-invariant subspace of the 3-dimensional space of aQGC operators containing only covariant derivatives, and thus directly translatable into our setting. However, they are weaker than the ones we have obtained, and they are not shown in figure 8 .

\subsection{Positivity bounds}

The space of Wilson coefficients can also be constrained theoretically by imposing general principles such as unitarity, locality and causality. The bounds obtained in this way are known as positivity bounds [37], and can be interpreted as necessary conditions for the existence of a UV completion to the EFT in question. In the HEFT, causality implies that [38-41]

$$
c_{D 1,0}+c_{D 2,0}>0, \quad c_{D 2,0}>0 .
$$

These inequalities also arise in the chiral Lagrangian without gauge bosons [42]. The region excluded by them is shown in blue in figure 8. It follows that skyrmions can only exist 
in the angular region $\theta_{\min } \leq \theta<3 \pi / 4$. Combining this fact with the experimental limits gives an upper bound on the mass of the skyrmion:

$$
M_{\mathrm{Sk}} \lesssim 1.6 \mathrm{TeV}
$$

\subsection{Dark matter}

Similarly to skyrmion production, skyrmion decay is a $B+L$-violating process which expected to be exponentially suppressed. The skyrmion lifetime is thus likely longer than the age of the universe, opening the possibility of skyrmions being Dark Matter (DM) candidates. We use the following order-of-magnitude estimate of the freeze-out skyrmion density $[6]$

$$
\Omega_{\mathrm{Sk}} h^{2} \simeq \frac{3 \times 10^{-27} \mathrm{~cm}^{3} \mathrm{~s}^{-1}}{\left\langle\sigma_{\mathrm{ann} \mathrm{V}}\right\rangle}, \quad \sigma_{\mathrm{ann}} \simeq \pi R_{\mathrm{Sk}}^{2}, \quad \mathrm{v} \simeq 1 / 2 .
$$

Requiring that the skyrmion density is at most the total DM density $\Omega_{\mathrm{Sk}} h^{2} \simeq 0.1$ gives a skyrmion mass of $M_{\mathrm{Sk}} \simeq 60 \mathrm{GeV}$. This naive estimate is well below the lower limit induced the requirement that the skyrmions behave classically, making them not viable as DM candidates. However, more sophisticated calculations have to be performed to see if a skyrmion with mass above this limit can satisfy the abundance criterion. The simple scenario we have considered can be substantially modified by effects such as the possible existence of long-range interactions between skyrmions and antiskyrmions, or the dependence of the skyrmion mass and lifetime on temperature. A detailed exploration of these topics is beyond the scope of this paper and deserves its own study.

\section{Conclusions}

We have studied the skyrmion configurations that arise in the HEFT. We have found that a metastable configuration with classical behavior and skyrmion number close to one exists whenever the coefficients $c_{D 1,0}$ and $c_{D 2,0}$ lie on the strip

$$
c_{D 2,0} \leq \tan \left(\theta_{\min }\right) c_{D 1,0}, \quad 1.7 \times 10^{-3} \lesssim \tan \left(\theta_{\max }\right) c_{D 1,0}-c_{D 2,0} \lesssim 0.13 .
$$

The mass of this skyrmion is given by $M_{\mathrm{Sk}}=(30 \mathrm{TeV}) \cdot\left[\tan \left(\theta_{\max }\right) c_{D 1,0}-c_{D 2,0}\right]^{1 / 2}$. It is separated from the trivial vacuum by an energy barrier of about $11 \mathrm{TeV}$. This value also represents the maximal theoretical $M_{\mathrm{Sk}}$, as above it the barrier disappears. The minimal mass is obtained by requiring that the Compton wavelength of the skyrmion is shorter than its size, which gives $M_{\mathrm{Sk}} \gtrsim 1.2 \mathrm{TeV}$.

Since skyrmions are unlikely to be created at colliders, we have focused on the experimental signals of the operators that stabilize them. LHC searches for aQCG put bounds of order $10^{-3}$ on both coefficients. Combining these bounds with positivity constraints, we have found that the allowed parameter space for skyrmions in the quadrilateral

$$
\begin{aligned}
1<c_{D 2,0} / c_{D 1,0} & \leq \tan \left(\theta_{\min }\right), \quad c_{D 1,0}>-1.3 \times 10^{-3}, \\
c_{D 2,0} & \gtrsim 1.7 \times 10^{-3} \tan \left(\theta_{\max }\right) c_{D 1,0} .
\end{aligned}
$$


This allowed us to obtain a stronger upper bound on the mass of the skyrmion, of about $1.6 \mathrm{TeV}$.

Skyrmions are also expected to be long-lived, so they contribute to the DM density. A naive estimate of their abundance in a freeze-out scenario shows that the required $M_{\mathrm{Sk}}$ for all the DM to be made of skyrmions is outside the range in which they have a classical behavior. However, more detailed calculations of their annihilation cross section and of the effects of finite temperature are needed to determine their viability as DM candidates.

\section{A Numerical method}

We use a neural network to model the function taking $r$ to $R(r)=\left(f_{1}(r), f_{2}(r), b(r), \eta(r)\right)$. We choose an architecture with sigmoid $\sigma(x)=\left(1+e^{-x}\right)^{-1}$ activation functions and two hidden layers, each having 5 units. That is, we parameterize

$$
R(r)=A_{3} \circ \sigma \circ A_{2} \circ \sigma \circ A_{1}(r)
$$

where $A_{1}: \mathbb{R}^{1} \rightarrow \mathbb{R}^{5}, A_{2}: \mathbb{R}^{5} \rightarrow \mathbb{R}^{5}$, and $A_{3}: \mathbb{R}^{5} \rightarrow \mathbb{R}^{4}$ are affine transformations. The problem is to adjust the parameters of $A_{1}, A_{2}$ and $A_{3}$ to minimize the energy $E[R]$ while satisfying the boundary conditions $\mathrm{BC}[R]=0$ and fixing $n_{W}[R]$ to some value $n_{W, 0}$. We reformulate this problem as minimizing the functional

$$
L[R]=E[R]+\omega_{\mathrm{BC}} \mathrm{BC}[R]^{2}+\omega_{n_{W}}\left(n_{W}[R]-n_{W, 0}\right)^{2},
$$

with the weights $\omega_{i}$ being sufficiently high. In practice, we set $\omega_{\mathrm{BC}}=10^{3}$ and $\omega_{n_{W}}=10^{4}$. To perform the numerical minimization of $L$, the training of the network, we use the functional-minimization features of Elvet [43, 44].

This procedure allows us to find the minimal energy $E$ for a fixed $n_{W}$. In order to find the local minimum near $n_{W}=1$, which is the skyrmion, we first train the network with $n_{W}=1$. Once the minimum under this condition is reached, we resume the training with this condition removed by setting $\omega_{n_{W}}=0$. Since the network is already close to the local minimum, the training cannot overcome the finite barrier, so it can only take the network into the skyrmion configuration.

We check that the configurations found in this way are compatible with Derrick's argument. We first split the energy as

$$
E=\sum_{i} E_{i}, \quad E_{i}=\frac{4 \pi v}{e} \int_{0}^{\infty} d r F_{i}(\eta) \rho_{i} .
$$

In terms of the functions defined here, a spatial scale transformation is given as $r \rightarrow \lambda r$, $b \rightarrow b / \lambda$. If one applies such transformation to a local minimum, the energy must satisfy:

$$
0=\left.\frac{d E}{d \lambda}\right|_{\lambda=1}=3 E_{1}+\left(E_{h}+E_{U}\right)-\sum_{i} E_{i},
$$

where $i$ runs over rest of operators in table 1 . Numerically, we get

$$
\left.\frac{1}{E} \frac{d E}{d \lambda}\right|_{\lambda=1}<1 \%
$$


In order to find the critical value $e_{\text {crit }}$, we note that for $e>e_{\text {crit }}$ the height of the local minimum must be lower than the height of the barrier, so at $e=e_{\text {crit }}$ they must become of the same height. For small $e$, the barrier is found at around $n_{W}=0.4$ and the local minimum is close to $n_{W}=0.8$. We can thus obtain $e_{\text {crit }}$ approximately as the value of $e$ that minimizes $\left|E\left(n_{W}=0.4\right)-E\left(n_{W}=0.8\right)\right|$. We search for this value in steps of 0.05 .

Open Access. This article is distributed under the terms of the Creative Commons Attribution License (CC-BY 4.0), which permits any use, distribution and reproduction in any medium, provided the original author(s) and source are credited.

\section{References}

[1] T.H.R. Skyrme, A nonlinear field theory, Proc. Roy. Soc. Lond. A 260 (1961) 127 [inSPIRE].

[2] E. Witten, Baryons in the 1/n expansion, Nucl. Phys. B 160 (1979) 57 [InSPIRE].

[3] G.S. Adkins, C.R. Nappi and E. Witten, Static properties of nucleons in the Skyrme model, Nucl. Phys. B 228 (1983) 552 [inSPIRE].

[4] J. Ellis, M. Karliner and M. Praszalowicz, Generalized skyrmions in $Q C D$ and the electroweak sector, JHEP 03 (2013) 163 [arXiv:1209.6430] [INSPIRE].

[5] J. Ambjørn and V.A. Rubakov, Classical versus semiclassical electroweak decay of a techniskyrmion, Nucl. Phys. B 256 (1985) 434 [INSPIRE].

[6] J.C. Criado, V.V. Khoze and M. Spannowsky, The emergence of electroweak skyrmions through Higgs bosons, JHEP 03 (2021) 162 [arXiv:2012.07694] [INSPIRE].

[7] G.H. Derrick, Comments on nonlinear wave equations as models for elementary particles, J. Math. Phys. 5 (1964) 1252 [InSPIRE].

[8] ATLAS collaboration, Observation of a new particle in the search for the Standard Model Higgs boson with the ATLAS detector at the LHC, Phys. Lett. B 716 (2012) 1 [arXiv: 1207.7214] [INSPIRE].

[9] CMS collaboration, Observation of a new boson at a mass of $125 \mathrm{GeV}$ with the CMS experiment at the LHC, Phys. Lett. B $\mathbf{7 1 6}$ (2012) 30 [arXiv:1207.7235] [INSPIRE].

[10] S.R. Coleman, J. Wess and B. Zumino, Structure of phenomenological Lagrangians. 1, Phys. Rev. 177 (1969) 2239 [INSPIRE].

[11] C.G. Callan, Jr., S.R. Coleman, J. Wess and B. Zumino, Structure of phenomenological Lagrangians. 2, Phys. Rev. 177 (1969) 2247 [INSPIRE].

[12] F. Feruglio, The chiral approach to the electroweak interactions, Int. J. Mod. Phys. A 8 (1993) 4937 [hep-ph/9301281] [INSPIRE].

[13] C.P. Burgess, J. Matias and M. Pospelov, A Higgs or not a Higgs? What to do if you discover a new scalar particle, Int. J. Mod. Phys. A 17 (2002) 1841 [hep-ph/9912459] [inSPIRE].

[14] B. Grinstein and M. Trott, A Higgs-Higgs bound state due to new physics at a TeV, Phys. Rev. D 76 (2007) 073002 [arXiv:0704.1505] [INSPIRE].

[15] R. Contino, C. Grojean, M. Moretti, F. Piccinini and R. Rattazzi, Strong double Higgs production at the LHC, JHEP 05 (2010) 089 [arXiv:1002.1011] [INSPIRE]. 
[16] R. Alonso, M.B. Gavela, L. Merlo, S. Rigolin and J. Yepes, The effective chiral Lagrangian for a light dynamical "Higgs particle", Phys. Lett. B $\mathbf{7 2 2}$ (2013) 330 [Erratum ibid. $\mathbf{7 2 6}$ (2013) 926] [arXiv: 1212.3305] [INSPIRE].

[17] G. Buchalla, O. Catà and C. Krause, Complete electroweak chiral Lagrangian with a light Higgs at NLO, Nucl. Phys. B 880 (2014) 552 [Erratum ibid. 913 (2016) 475] [arXiv: 1307.5017] [INSPIRE].

[18] G. Buchalla, M. Capozi, A. Celis, G. Heinrich and L. Scyboz, Higgs boson pair production in non-linear effective field theory with full $m_{t}$-dependence at NLO QCD, JHEP 09 (2018) 057 [arXiv: 1806.05162] [INSPIRE].

[19] A. Manohar and H. Georgi, Chiral quarks and the nonrelativistic quark model, Nucl. Phys. B 234 (1984) 189 [INSPIRE].

[20] A. Pich, I. Rosell, J. Santos and J.J. Sanz-Cillero, Fingerprints of heavy scales in electroweak effective Lagrangians, JHEP 04 (2017) 012 [arXiv: 1609.06659] [INSPIRE].

[21] R. Kitano and M. Kurachi, Electroweak-skyrmion as topological dark matter, JHEP 07 (2016) 037 [arXiv : 1605.07355] [INSPIRE].

[22] R. Kitano and M. Kurachi, More on electroweak-skyrmion, JHEP 04 (2017) 150 [arXiv: 1703.06397] [INSPIRE].

[23] Y. Hamada, R. Kitano and M. Kurachi, Electroweak-skyrmion as asymmetric dark matter, arXiv:2108.12185 [INSPIRE].

[24] S. Das Bakshi, J. Chakrabortty, C. Englert, M. Spannowsky and P. Stylianou, CP violation at ATLAS in effective field theory, Phys. Rev. D 103 (2021) 055008 [arXiv:2009.13394] [INSPIRE].

[25] T. Banks, G.R. Farrar, M. Dine, D. Karabali and B. Sakita, Weak interactions are weak at high-energies, Nucl. Phys. B 347 (1990) 581 [inSPIRE].

[26] CMS collaboration, Study of vector boson scattering and search for new physics in events with two same-sign leptons and two jets, Phys. Rev. Lett. 114 (2015) 051801 [arXiv: 1410.6315] [INSPIRE].

[27] ATLAS collaboration, Evidence of $W \gamma \gamma$ production in pp collisions at $\sqrt{s}=8 \mathrm{TeV}$ and limits on anomalous quartic gauge couplings with the ATLAS detector, Phys. Rev. Lett. 115 (2015) 031802 [arXiv: 1503.03243] [INSPIRE].

[28] ATLAS collaboration, Studies of $Z \gamma$ production in association with a high-mass dijet system in pp collisions at $\sqrt{s}=8 \mathrm{TeV}$ with the ATLAS detector, JHEP 07 (2017) 107 [arXiv: 1705. 01966] [INSPIRE].

[29] ATLAS collaboration, Study of $W W \gamma$ and $W Z \gamma$ production in pp collisions at $\sqrt{s}=8 \mathrm{TeV}$ and search for anomalous quartic gauge couplings with the ATLAS experiment, Eur. Phys. J. C 77 (2017) 646 [arXiv: 1707.05597] [INSPIRE].

[30] CMS collaboration, Search for anomalous electroweak production of vector boson pairs in association with two jets in proton-proton collisions at 13 TeV, Phys. Lett. B 798 (2019) 134985 [arXiv: 1905.07445] [INSPIRE].

[31] CMS collaboration, Measurements of production cross sections of $W Z$ and same-sign $W W$ boson pairs in association with two jets in proton-proton collisions at $\sqrt{s}=13 \mathrm{TeV}$, Phys. Lett. B 809 (2020) 135710 [arXiv: 2005.01173] [INSPIRE]. 
[32] CMS collaboration, Evidence for electroweak production of four charged leptons and two jets in proton-proton collisions at $\sqrt{s}=13 \mathrm{TeV}$, Phys. Lett. B 812 (2021) 135992 [arXiv: 2008.07013] [INSPIRE].

[33] O.J.P. Eboli, M.C. Gonzalez-Garcia and J.K. Mizukoshi, $p p \rightarrow j j e^{ \pm} \mu^{ \pm} \nu \nu$ and $j j e^{ \pm} \mu^{\mp} \nu \nu$ at $O\left(\alpha_{\mathrm{em}}^{6}\right)$ and $O\left(\alpha_{\mathrm{em}}^{4} \alpha_{s}^{2}\right)$ for the study of the quartic electroweak gauge boson vertex at CERN LHC, Phys. Rev. D 74 (2006) 073005 [hep-ph/0606118] [INSPIRE].

[34] O.J.P. Éboli and M.C. Gonzalez-Garcia, Classifying the bosonic quartic couplings, Phys. Rev. D 93 (2016) 093013 [arXiv: 1604.03555] [INSPIRE].

[35] M. Rauch, Vector-boson fusion and vector-boson scattering, arXiv:1610.08420 [INSPIRE].

[36] ATLAS collaboration, Search for anomalous electroweak production of $W W / W Z$ in association with a high-mass dijet system in pp collisions at $\sqrt{s}=8 \mathrm{TeV}$ with the ATLAS detector, Phys. Rev. D 95 (2017) 032001 [arXiv:1609.05122] [InSPIRE].

[37] A. Adams, N. Arkani-Hamed, S. Dubovsky, A. Nicolis and R. Rattazzi, Causality, analyticity and an IR obstruction to UV completion, JHEP 10 (2006) 014 [hep-th/0602178] [INSPIRE].

[38] J. Distler, B. Grinstein, R.A. Porto and I.Z. Rothstein, Falsifying models of new physics via WW scattering, Phys. Rev. Lett. 98 (2007) 041601 [hep-ph/0604255] [INSPIRE].

[39] M. Fabbrichesi, M. Pinamonti, A. Tonero and A. Urbano, Vector boson scattering at the LHC: a study of the $W W \rightarrow W W$ channels with the Warsaw cut, Phys. Rev. D 93 (2016) 015004 [arXiv: 1509.06378] [INSPIRE].

[40] C. Zhang and S.-Y. Zhou, Positivity bounds on vector boson scattering at the LHC, Phys. Rev. D 100 (2019) 095003 [arXiv: 1808.00010] [INSPIRE].

[41] Q. Bi, C. Zhang and S.-Y. Zhou, Positivity constraints on aQGC: carving out the physical parameter space, JHEP 06 (2019) 137 [arXiv: 1902.08977] [INSPIRE].

[42] A. Jenkins and D. O'Connell, The story of O: positivity constraints in effective field theories, hep-th/0609159 [INSPIRE].

[43] J.Y. Araz, J.C. Criado and M. Spannowsky, Elvet - a neural network-based differential equation and variational problem solver, arXiv:2103.14575 [INSPIRE].

[44] M.L. Piscopo, M. Spannowsky and P. Waite, Solving differential equations with neural networks: applications to the calculation of cosmological phase transitions, Phys. Rev. D 100 (2019) 016002 [arXiv: 1902.05563] [INSPIRE]. 